\title{
Approximation on parametric extension of Baskakov-Durrmeyer operators on weighted spaces
}

\author{
Md Nasiruzzaman ${ }^{\text {* }}$, Nadeem Rao², Samar Wazir ${ }^{1}$ and Ravi Kumar ${ }^{3}$
}

\section{"Correspondence:}

nasir3489@gmail.com

'Department of Computer Science

(SEST), Jamia Hamdard University,

New Delhi, India

Full list of author information is

available at the end of the article

\section{Springer}

\begin{abstract}
In the present manuscript, we define a non-negative parametric variant of Baskakov-Durrmeyer operators to study the convergence of Lebesgue measurable functions and introduce these as $\alpha$-Baskakov-Durrmeyer operators. We study the uniform convergence of these operators in weighted spaces.

MSC: Primary 41A25; 41A36; secondary 33C45

Keywords: Baskakov operators; Simultaneous approximation; Korovkin-type approximation theorems; Weighted modulus of continuity; Rate of convergence; Peetre's K-functional
\end{abstract}

\section{Introduction}

In the field of mathematical analysis, Karl Weierstrass established an elegant theorem, the first Weierstrass approximation theorem, in 1885. This theorem has specially a big role in polynomial interpolation corresponding to every continuous function $f(x)$ on interval $[a, b]$. The proof given by Weierstrass was rigorous and difficult to understand. In 1912, Bernstein [1] gave a simple proof of this theorem by introducing the Bernstein polynomials with the aid of the binomial distribution, hence for $f \in C[0,1]$, we have

$$
B_{n}(f ; x)=\sum_{k=0}^{n} \mathcal{S}_{n, k}(x) f\left(\frac{k}{n}\right), \quad n \in \mathbb{N}, 0 \leq x \leq 1,
$$

where $\mathcal{S}_{n, k}(x)=\left(\begin{array}{l}n \\ k\end{array}\right) x^{k}(1-x)^{n-k}$. Many mathematicians researched in this direction and studied various modifications in several functional spaces using different error optimization techniques, i.e., Acar et al. [2-7], Acu et al. [8, 9], Barbosu [10], Agrawal et al. [11], Aral [12], Mursaleen et al. [13-17], Srivastava et al. [18-20]; for more details see also the references therein and [21-30].

\section{Construction of the $\alpha$-Baskakov-Durrmeyer operators and estimation of their moments}

Recently, Cai, Lian and Zhou [31] presented a new sequence of $\alpha$-Bernstein operators with $\alpha \in[-1,1]$. Later, Ali Aral et al. [32] gave a sequence of $\alpha$-Bernstein operators as

(c) The Author(s) 2019. This article is distributed under the terms of the Creative Commons Attribution 4.0 International License (http://creativecommons.org/licenses/by/4.0/), which permits unrestricted use, distribution, and reproduction in any medium, provided you give appropriate credit to the original author(s) and the source, provide a link to the Creative Commons license, and indicate if changes were made. 
follows:

$$
L_{n, \alpha}(f ; x)=\sum_{k=0}^{\infty} f\left(\frac{k}{n}\right) \mathcal{S}_{n, k}^{(\alpha)}(x), \quad n \in \mathbb{N}, x \in[0, \infty)
$$

where $f \in C_{B}[0, \infty)$ which denotes the set of all continuous and bounded functions and

$$
\begin{aligned}
\mathcal{S}_{n, k}^{(\alpha)}(x)= & \frac{x^{k-1}}{(1+x)^{n+k-1}}\left\{\frac{\alpha x}{1+x}\left(\begin{array}{c}
n+k-1 \\
k
\end{array}\right)-(1-\alpha)(1+x)\left(\begin{array}{c}
n+k-3 \\
k-2
\end{array}\right)\right. \\
& \left.+(1-\alpha) y\left(\begin{array}{c}
n+k-1 \\
k
\end{array}\right)\right\}
\end{aligned}
$$

with

$$
\left(\begin{array}{c}
n-3 \\
-2
\end{array}\right)=\left(\begin{array}{c}
n-2 \\
-1
\end{array}\right)=0
$$

The operators defined by (2.1) are restricted for continuous functions only. To approximate the functions in Lebesgue measurable space, we design a new sequence of operators:

$$
L_{n, \alpha}^{*}(f ; x)=\sum_{k=0}^{\infty} \mathcal{S}_{n, k}^{(\alpha)}(x) \int_{0}^{\infty} \mathcal{Q}_{n, k}(t) f(t) d t
$$

where $\mathcal{Q}_{n, k}(t)=\frac{1}{B(k+1, n)} \frac{t^{k}}{(1+t)^{(n+k+1)}}$. Note that, simply in the case of $\alpha=1$, the operators reduced to Baskakov-Durrmeyer type operators; for details see [33].

For $r \in\{0,1,2,3,4\}$, we consider the test functions and central moments,

$$
e_{r}=t^{r} \quad \text { and } \quad \psi_{y}^{r}(t ; x)=(t-x)^{r} .
$$

Lemma 2.1 ([31]) We have

$$
\begin{aligned}
& L_{n, \alpha}\left(e_{0} ; x\right)=1, \\
& L_{n, \alpha}\left(e_{1} ; x\right)=x+\frac{2}{n}(\alpha-1), \\
& L_{n, \alpha}\left(e_{2} ; x\right)=x^{2}+\frac{4 \alpha-3}{n} x+\frac{1}{n^{2}}(n+4 \alpha-4) .
\end{aligned}
$$

Lemma 2.2 Let the test functions $e_{r}$ defined by (2.3), then, for all $L_{n, \alpha}^{*}$, we have

$$
\begin{aligned}
& L_{n, \alpha}^{*}\left(e_{0} ; x\right)=1 \\
& L_{n, \alpha}^{*}\left(e_{1} ; x\right)=\left(\frac{n}{n-1}+\frac{2(\alpha-1)}{n-1}\right) x+\frac{1}{n-1}, \\
& L_{n, \alpha}^{*}\left(e_{2} ; x\right)=\left(\frac{n^{2}}{(n-2)(n-1)}+\frac{n(4 \alpha-3)}{(n-2)(n-1)}\right) x^{2}+\frac{(4 n+10 \alpha-10)}{(n-2)(n-1)} x+\frac{2}{(n-2)(n-1)} .
\end{aligned}
$$


Proof Take $f=e_{0}$, then from Lemma 2.1, we have

$$
\begin{aligned}
L_{n, \alpha}^{*}\left(e_{0} ; x\right) & =\sum_{k=0}^{\infty} \mathcal{S}_{n, k}^{(\alpha)}(x) \int_{0}^{\infty} \mathcal{Q}_{n, k}(t) d t \\
& =\sum_{k=0}^{\infty} \mathcal{S}_{n, k}^{(\alpha)}(x) \frac{B(k+1, n)}{B(k+1, n)} \\
& =\sum_{k=0}^{\infty} \mathcal{S}_{n, k}^{(\alpha)}(x) \\
& =1 .
\end{aligned}
$$

For $r=1$

$$
\begin{aligned}
L_{n, \alpha}^{*}\left(e_{1} ; x\right) & =\sum_{k=0}^{\infty} \mathcal{S}_{n, k}^{(\alpha)}(x) \int_{0}^{\infty} t \mathcal{Q}_{n, k}(t) d t \\
& =\sum_{k=0}^{\infty} \mathcal{S}_{n, k}^{(\alpha)}(x) \frac{B(k+2, n-1)}{B(k+1, n)} \\
& =\sum_{k=0}^{\infty} \mathcal{S}_{n, k}^{(\alpha)}(x) \frac{(k+1) B(k+1, n)}{(n-1) B(k+1, n)} \\
& =\sum_{k=0}^{\infty} \mathcal{S}_{n, k}^{(\alpha)}(x) \frac{(k+1)}{(n-1)} \\
& =\left(\frac{n}{n-1}+\frac{2(\alpha-1)}{n-1}\right) x+\frac{1}{n-1}
\end{aligned}
$$

For $r=2$

$$
\begin{aligned}
L_{n, \alpha}^{*}\left(e_{2} ; x\right) & =\sum_{k=0}^{\infty} \mathcal{S}_{n, k}^{(\alpha)}(x) \int_{0}^{\infty} t^{2} \mathcal{Q}_{n, k}(t) d t \\
& =\sum_{k=0}^{\infty} \mathcal{S}_{n, k}^{(\alpha)}(x) \frac{B(k+3, n-2)}{B(k+1, n)} \\
& =\sum_{k=0}^{\infty} \mathcal{S}_{n, k}^{(\alpha)}(x) \frac{(k+2)(k+1) B(k+1, n)}{(n-2)(n-1) B(k+1, n)} \\
& =\sum_{k=0}^{\infty} \mathcal{S}_{n, k}^{(\alpha)}(x) \frac{(k+2)(k+1)}{(n-2)(n-1)} \\
& =\frac{n^{2}+n(4 \alpha-3)}{(n-2)(n-1)} x^{2}+\frac{(4 n+10 \alpha-10)}{(n-2)(n-1)} x+\frac{2}{(n-2)(n-1)}
\end{aligned}
$$

Lemma 2.3 Let the operators given by (2.2). Then we have

$$
\begin{aligned}
& L_{n, \alpha}^{*}\left(\psi_{x}^{0} ; x\right)=1 \\
& L_{n, \alpha}^{*}\left(\psi_{x}^{1} ; x\right)=\frac{2 \alpha-1}{n-1} x+\frac{1}{n-1},
\end{aligned}
$$




$$
L_{n, \alpha}^{*}\left(\psi_{x}^{2} ; x\right)=\frac{2 n+2(4 \alpha-3)}{(n-2)(n-1)} x^{2}+\frac{2 n+2(5 \alpha-3)}{(n-2)(n-1)} x+\frac{2}{(n-2)(n-1)} .
$$

Proof In view of Lemmas 2.1 and 2.2 we can apply the linearity and easily complete the proof.

\section{Approximation in Korovkin and weighted Korovkin spaces}

Take $C_{B}\left(\mathbb{R}^{+}\right)$be the space of all bounded and continuous functions defined on the set $\mathbb{R}^{+}$, where $\mathbb{R}^{+}=[0, \infty)$ and a normed defined on $C_{B}$ as

$$
\|f\|_{C_{B}}=\sup _{x \geq 0}|f(x)|
$$

Let

$$
E:=\left\{f: x \in \mathbb{R}^{+} \text {and } \lim _{x \rightarrow \infty}\left(\frac{f(x)}{1+x^{2}}\right)<\infty\right\} .
$$

Lemma 3.1 For every $f \in C[0, \infty) \cap E$ the operators $L_{n, \alpha}^{*}$ given in (2.2) are uniformly convergent to $f$ on each compact subset of $[0, A]$, whenever $A \in(0, \infty)$.

Proof In the view of Korovkin-type property, it is enough to show that

$$
L_{n, \alpha}^{*}\left(e_{s} ; x\right) \rightarrow e_{s}(x), \quad \text { for } s=0,1,2 .
$$

From Lemma 2.2, obviously $L_{n, \alpha}^{*}\left(e_{0} ; y\right) \rightarrow e_{0}(x)$ as $n \rightarrow \infty$ and for $s=1$

$$
\lim _{n \rightarrow \infty} L_{n, \alpha}^{*}\left(e_{1} ; x\right)=\lim _{n \rightarrow \infty}\left(\frac{n+2(\alpha-1)}{n-1} x+\frac{1}{n-1}\right)=e_{1}(x) .
$$

Similarly, we can prove for $s=2$ that $L_{n, \alpha}^{*}\left(e_{2} ; x\right) \rightarrow e_{2}$, which proves Proposition 3.1.

Suppose $C[0, \infty)$ is the set of all continuous functions and $f \in C[0, \infty)$ with the weight function $\sigma(x)=1+x^{2}$,

$$
\begin{aligned}
& \mathfrak{P}_{\sigma}(x)=\left\{f:|f(x)| \leq \mathcal{M}_{f} \sigma(x), x \in[0, \infty)\right\}, \\
& \mathfrak{Q}_{\sigma}(x)=\left\{f: f \in C[0, \infty) \cap \mathfrak{P}_{\sigma}(x), x \in[0, \infty)\right\}, \\
& \mathfrak{Q}_{\sigma}^{m}(x)=\left\{f: f \in \mathfrak{Q}_{\sigma}(x), \lim _{x \rightarrow \infty} \frac{f(x)}{\sigma(x)}=m, x \in[0, \infty)\right\},
\end{aligned}
$$

where the norm defined on weight function $\sigma$ such as $\|f\|_{\sigma}=\sup _{x \in[0, \infty)} \frac{|f(x)|}{\sigma(x)}$ and the constant $\mathcal{M}_{f}$ depends only on $f$.

Theorem 3.2 For all $f \in \mathfrak{Q}_{\sigma}^{m}(x)$ the operators $L_{n, \alpha}^{*}(\cdot ; \cdot)$ defined by (2.2) satisfy

$$
\lim _{n \rightarrow \infty}\left\|L_{n, \alpha}^{*}(f ; x)-f\right\|_{\sigma}=0 .
$$


Proof Take $f(t) \in \mathfrak{Q}_{\sigma}^{m}(x)$ with $x \in[0, \infty)$ and $f(t)=e_{v}$ for $v=0,1,2$. Then from the wellknown Korovkin theorem $L_{n, \alpha}^{*}\left(e_{\nu} ; x\right) \rightarrow x^{\nu}$, satisfying the properties of uniformly behaving as $n \rightarrow \infty$. Since for $v=0$, from Lemma $2.2 L_{n, \alpha}^{*}\left(e_{0} ; x\right)=1$, thus we have

$$
\left\|L_{n, \alpha}^{*}\left(e_{0} ; x\right)-1\right\|_{\sigma}=0 .
$$

For $v=1$, we have

$$
\begin{aligned}
\left\|L_{n, \alpha}^{*}\left(e_{1} ; x\right)-x\right\|_{\sigma} & =\sup _{x \in[0, \infty)} \frac{\left|L_{n, \alpha}^{*}\left(e_{1} ; x\right)-x\right|}{1+x^{2}} \\
& =\left(\frac{n+2(\alpha-1)}{n-1}-1\right) \sup _{x \in[0, \infty)} \frac{x}{1+x^{2}}+\frac{1}{(n-1)} \sup _{x \in[0, \infty)} \frac{1}{1+x^{2}} .
\end{aligned}
$$

As $n \rightarrow \infty$,

$$
\left\|L_{n, \alpha}^{*}\left(e_{1} ; x\right)-x\right\|_{\sigma}=0 .
$$

In a similar way for $v=2$,

$$
\begin{aligned}
& \left\|L_{n, \alpha}^{*}\left(e_{2} ; x\right)-x^{2}\right\|_{\sigma} \\
& \quad=\sup _{y \in[0, \infty)} \frac{\left|L_{n, \alpha}^{*}\left(e_{2} ; x\right)-x^{2}\right|}{1+x^{2}} \\
& \quad=\left(\frac{n^{2}+n(4 \alpha-3)}{(n-2)(n-1)}-1\right) \sup _{x \in[0, \infty)} \frac{x^{2}}{1+x^{2}} \\
& \quad+\left(\frac{4 n+10 \alpha-10}{(n-2)(n-1)}\right) \sup _{x \in[0, \infty)} \frac{x}{1+x^{2}}+\frac{2}{(n-2)(n-1)} \sup _{x \in[0, \infty)} \frac{1}{1+x^{2}}, \\
& \left\|L_{n, \alpha}^{*}\left(e_{2} ; x\right)-x^{2}\right\|_{\sigma}=0 \quad \text { when } n \rightarrow \infty .
\end{aligned}
$$

This completes the proof.

\section{Pointwise approximation properties by $L_{n, \alpha}^{*}$}

Here, we study the order of approximation of a function $f$ with the aid of positive linear operators $L_{n, \alpha}^{*}(f ; x)$ defined by $(2.2)$ in terms of the classical modulus of continuity, the second-order modulus of continuity, Peetres $K$-functional and the Lipschitz class. A wellknown property is the modulus of continuity of order one and of order two defined as follows. For $\delta>0$ and $f \in C[a, b]$ the classical modulus of continuity of order one is given by

$$
\omega(f ; \delta)=\sup _{x_{1}, x_{2} \in[a, b],\left|x_{1}-x_{2}\right| \leq \delta}\left|f\left(x_{1}\right)-f\left(x_{2}\right)\right|
$$

and of order two it is given by

$$
\omega_{2}\left(f ; \delta^{\frac{1}{2}}\right)=\sup _{0<h<\delta} \sup _{\substack{\frac{1}{2} \\ x \in \mathbb{R}^{+}}}|f(x)-2 f(x+h)+f(x+2 h)| .
$$


Let $C_{B}[0, \infty)$ denote the space of all bounded and continuous functions on $[0, \infty)$ and

$$
C_{B}^{2}[0, \infty)=\left\{\psi \in C_{B}[0, \infty): \psi^{\prime}, \psi^{\prime \prime} \in C_{B}[0, \infty)\right\}
$$

with the norm

$$
\|\psi\|_{C_{B}^{2}[0, \infty)}=\|\psi\|_{C_{B}[0, \infty)}+\left\|\psi^{\prime}\right\|_{C_{B}[0, \infty)}+\left\|\psi^{\prime \prime}\right\|_{C_{B}[0, \infty)},
$$

also

$$
\|\psi\|_{C_{B}[0, \infty)}=\sup _{x \in[0, \infty)}|\psi(x)| .
$$

Lemma 4.1 ([31]) Let $\left\{P_{n}\right\}_{n \geq 1}$ be the sequence for the positive integer $n$ with $P_{n}(1 ; x)=1$. Then for every $\psi \in C_{B}^{2}[0, \infty)$

$$
\left|P_{n}(\psi ; x)-\psi(x)\right| \leq\left\|g^{\prime}\right\| \sqrt{P_{n}\left((s-x)^{2} ; x\right)}+\frac{1}{2}\left\|\psi^{\prime \prime}\right\| P_{n}\left((s-x)^{2} ; x\right) .
$$

Lemma 4.2 ([31]) For all $f \in C[a, b]$ and $h \in\left(0, \frac{b-a}{2}\right)$, we have the following inequalities:

$$
\begin{aligned}
& \text { (i) }\left\|f_{h}-f\right\| \leq \frac{3}{4} \omega_{2}(f, h), \\
& \text { (ii) }\left\|f_{h}^{\prime \prime}\right\| \leq \frac{3}{2 h^{2}} \omega_{2}(f, h),
\end{aligned}
$$

where $f_{h}$ denotes the second-order Steklov function.

Theorem 4.3 For all $f \in C_{B}[0, \infty)$ and $x \in[0, a], a>0$ we have

$$
\left|L_{n, \alpha}^{*}(f ; x)-f(x)\right| \leq 2 \omega\left(f ; \sqrt{\Theta_{n}(x)}\right)
$$

where $\Theta_{n}(x)=L_{n, \alpha}^{*}\left(\psi_{x}^{2} ; x\right)$ and $L_{n, \alpha}^{*}\left(\psi_{x}^{2} ; x\right)$ is defined by Lemma 2.3.

Proof In view of the classical modulus of continuity, we have

$$
\begin{aligned}
\left|L_{n, \alpha}^{*}(f ; x)-f(x)\right| & \leq \sum_{k=0}^{\infty} \mathcal{S}_{n, k}^{(\alpha)}(x) \int_{0}^{\infty} \mathcal{Q}_{n, k}(t)|f(t)-f(x)| d t \\
& \leq\left\{1+\frac{1}{\delta} \sum_{k=0}^{\infty} \mathcal{S}_{n, k}^{(\alpha)}(x) \int_{0}^{\infty} \mathcal{Q}_{n, k}(t)|t-x| d t\right\} \omega(f ; \delta) .
\end{aligned}
$$

In the light of the Cauchy-Schwartz inequality, we get

$$
\begin{aligned}
\left|L_{n, \alpha}^{*}(f ; x)-f(x)\right| & \leq\left\{1+\frac{1}{\delta}\left(\sum_{k=0}^{\infty} \mathcal{S}_{n, k}^{(\alpha)}(x) \int_{0}^{\infty} \mathcal{Q}_{n, k}(t)(t-x)^{2} d t\right)^{\frac{1}{2}}\right\} \omega(f ; \delta) \\
& =\left\{1+\frac{1}{\delta} \sqrt{L_{n, \alpha}^{*}\left(\psi_{x}^{2} ; x\right)}\right\} \omega(f ; \delta) .
\end{aligned}
$$

Choosing $\delta=\left(\Theta_{n}(x)\right)^{\frac{1}{2}}=\sqrt{L_{n, \alpha}^{*}\left(\psi_{x}^{2} ; x\right)}$, we arrive at the desired result. 
Theorem 4.4 For every $f \in C[0, a], a>0$ the operators $L_{n, \alpha}^{*}(\cdot ; \cdot)$ defined by (2.2) satisfy

$$
\left|L_{n, \alpha}^{*}(f ; x)-f(x)\right| \leq \frac{2}{a}\|f\| \delta^{2}+\frac{3}{4}\left(a+2+h^{2}\right) \omega_{2}(f ; \delta),
$$

where $\delta=\left(\Theta_{n}(x)\right)^{\frac{1}{2}}$ is defined by Theorem 4.3 and $\omega_{2}(f ; \delta)$ is by (4.1) equipped with the norm $\|f\|=\max _{x \in[a, b]}|f(x)|$.

Proof Consider $f_{h}$ is the Steklov function define in Lemma 4.2. Using Lemma 2.2, we obtain

$$
\begin{aligned}
\left|L_{n, \alpha}^{*}(f ; x)-f(x)\right| & \leq\left|L_{n, \alpha}^{*}\left(f-f_{h} ; x\right)\right|+\left|f_{h}-f(x)\right|+\left|L_{n, \alpha}^{*}\left(f_{h} ; x\right)-f_{h}(x)\right| \\
& \leq 2|| f_{h}-f \|+\left|L_{n, \alpha}^{*}\left(f_{h} ; x\right)-f_{h}(x)\right| .
\end{aligned}
$$

In view of the fact that $f_{h} \in C^{2}[0, a]$ and using Lemma 4.1, we obtain

$$
\left|L_{n, \alpha}^{*}(f ; x)-f(x)\right| \leq\left\|f_{h}^{\prime}\right\| \sqrt{L_{n, \alpha}^{*}\left(\left(e_{1}-x\right)^{2} ; x\right)}+\frac{1}{2}\left\|f_{h}^{\prime \prime}\right\| L_{n, \alpha}^{*}\left(\left(e_{1}-x\right)^{2} ; x\right) .
$$

From the Landau inequality and Lemma 4.2, we have

$$
\begin{aligned}
\left\|f_{h}\right\| & \leq \frac{2}{a}\left\|f_{h}\right\|+\frac{a}{2}\left\|f_{h}^{\prime \prime}\right\| \\
& \leq \frac{2}{a}\left\|f_{h}\right\|+\frac{3 a}{4} \frac{1}{h^{2}} \omega_{2}(f ; h) .
\end{aligned}
$$

On choosing $\delta=\left(\Theta_{n}(x)\right)^{\frac{1}{4}}$, one has

$$
\left|L_{n, \alpha}^{*}\left(f_{h} ; x\right)-f_{h}(x)\right| \leq \frac{2}{a}\|f\| h^{2}+\frac{3 a}{4} \omega_{2}(f ; h)+\frac{3}{4} h^{2} \omega_{2}(f ; h) .
$$

Combining (4.6), (4.5) and Lemma 4.2, we obtain the required result.

Theorem 4.5 Let $L_{n, \alpha}^{*}(\cdot ; \cdot)$ be the operators defined by (2.2). Then, for every $f \in C_{B}^{2}[0, \infty)$,

$$
\lim _{n \rightarrow \infty}(n-1)\left(L_{n, \alpha}^{*}(f ; x)-f(x)\right)=\left(1+2 \alpha x-x^{2}\right) f^{\prime}(x)+2\left(x+x^{2}\right) f^{\prime \prime}(x),
$$

uniformly for $0 \leq x \leq a, a>0$.

Proof Let $x_{0} \in[0, \infty)$ be a fixed number; all $x \in[0, \infty)$. Then using Taylor's series, we have

$$
f(x)-f\left(x_{0}\right)=\left(x-x_{0}\right) f^{\prime}\left(x_{0}\right)+\frac{1}{2}\left(x-x_{0}\right)^{2} f^{\prime \prime}\left(x_{0}\right)+\varphi\left(x, x_{0}\right)\left(x-x_{0}\right)^{2},
$$

where $\varphi\left(x, x_{0}\right) \in C_{B}[0, \infty)$ and $\lim _{x \rightarrow x_{0}} \varphi\left(x, x_{0}\right)=0$.

By applying the operators $L_{n, \alpha}^{*}$ on (4.7), we deduce

$$
\begin{aligned}
L_{n, \alpha}^{*}\left(f ; x_{0}\right)-f\left(x_{0}\right)= & f^{\prime}\left(x_{0}\right) L_{n, \alpha}^{*}\left(e_{1}-x_{0} ; x_{0}\right)+\frac{1}{2} L_{n, \alpha}^{*}\left(\left(x-x_{0}\right)^{2} ; x_{0}\right) f^{\prime \prime}\left(x_{0}\right) \\
& +L_{n, \alpha}^{*}\left(\varphi\left(x, x_{0}\right)\left(x-x_{0}\right)^{2}\right) .
\end{aligned}
$$


In view of the Cauchy-Schwartz inequality for the last term of Eq. (4.8), we get

$$
(n-1) L_{n, \alpha}^{*}\left(\varphi\left(x, x_{0}\right)\left(t-x_{0}\right)^{2}\right) \leq(n-1)^{2} \sqrt{L_{n, \alpha}^{*}\left(\left(e_{1}-x_{0}\right)^{2}\right) L_{n, \alpha}^{*}\left(\varphi^{2}\left(x, x_{0}\right)\right)} .
$$

We have

$$
\begin{aligned}
& \lim _{n \rightarrow \infty}(n-1)\left(L_{n, \alpha}^{*}\left(e_{0}-x_{0} ; x\right)\right)=\left(1+2 \alpha x-x^{2}\right) f^{\prime}(x), \\
& \lim _{n \rightarrow \infty}(n-1)\left(L_{n, \alpha}^{*}\left(\left(e_{0}-x_{0}\right)^{2} ; x\right)\right)=2\left(x+x^{2}\right) f^{\prime \prime}(x), \\
& \lim _{n \rightarrow \infty}\left(L_{n, \alpha}^{*}\left(\left(e_{0}-x_{0}\right)^{4} ; x\right)\right)=0 .
\end{aligned}
$$

This completes the proof.

Now here we estimate the rate of convergence in terms of the usual Lipschitz class $\operatorname{Lip}_{M}(v)$. Let $f \in C[0, a), a>0$ and $M$ be a positive constant, and, for any $v \in(0,1]$, the Lipschitz class $\operatorname{Lip}_{M}(v)$ is as follows:

$$
\operatorname{Lip}_{M}(v)=\left\{f:\left|f\left(\varsigma_{1}\right)-f\left(\varsigma_{2}\right)\right| \leq M\left|\varsigma_{1}-\varsigma_{2}\right|^{\nu}\left(\varsigma_{1}, \varsigma_{2} \in[0, \infty)\right)\right\}
$$

Theorem 4.6 Let $f \in \operatorname{Lip}_{M}(v)$ with $M>0$ and $0<v \leq 1$. Then the operators $L_{n, \alpha}^{*}(\cdot ; \cdot)$ satisfy

$$
\left|L_{n, \alpha}^{*}(f ; x)-f(x)\right| \leq M\left(\Theta_{n}(x)\right)^{\frac{v}{2}}
$$

where $n>2$ and $\Theta_{n}(x)$ defined by Theorem 4.3.

Proof From the Hölder inequality and (4.10), we conclude

$$
\begin{aligned}
\left|L_{n, \alpha}^{*}(f ; x)-f(x)\right| & \leq\left|L_{n, \alpha}^{*}(f(t)-f(x) ; x)\right| \\
& \leq L_{n, \alpha}^{*}(|f(t)-f(x)| ; x) \\
& \leq M L_{n, \alpha}^{*}\left(|t-x|^{\nu} ; x\right) .
\end{aligned}
$$

Hence

$$
\begin{aligned}
& \left|L_{n, \alpha}^{*}(f ; x)-f(x)\right| \\
& \leq M \sum_{k=0}^{\infty} \mathcal{S}_{n, k}^{(\alpha)}(x) \int_{0}^{\infty} \mathcal{Q}_{n, k}(t)|t-x|^{\nu} d t \\
& \leq M \sum_{k=0}^{\infty}\left(\mathcal{S}_{n, k}^{(\alpha)}(x)\right)^{\frac{2-v}{2}} \\
& \quad \times\left(\mathcal{S}_{n, k}^{(\alpha)}(x)\right)^{\frac{v}{2}} \int_{0}^{\infty} \mathcal{Q}_{n, k}(t)|t-x|^{\nu} d t \\
& \leq M\left(\sum_{k=0}^{\infty} \mathcal{S}_{n, k}^{(\alpha)}(x) \int_{0}^{\infty} \mathcal{Q}_{n, k}(t) d t\right)^{\frac{2-v}{2}}
\end{aligned}
$$




$$
\begin{aligned}
& \times\left(\sum_{k=0}^{\infty} \mathcal{S}_{n, k}^{(\alpha)}(x) \int_{0}^{\infty} \mathcal{Q}_{n, k}(t)|t-x|^{2} d t\right)^{\frac{v}{2}} \\
= & M\left(L_{n, \alpha}^{*}\left(\psi_{x}^{2} ; x\right)\right)^{\frac{v}{2}} .
\end{aligned}
$$

This completes the proof.

Theorem 4.7 For all $\psi \in C_{B}^{2}[0, \infty)$ and $n>2$,

$$
\left|L_{n, \alpha}^{*}(\psi ; x)-\psi(x)\right| \leq\left(\Delta_{n}(x)+\frac{\Theta_{n}(x)}{2}\right)\|\psi\|_{C_{B}^{2}[0, \infty)},
$$

where $\Delta_{n}(x)=\left(\frac{2 \alpha-1}{n-1} x+\frac{1}{n-1}\right)$ and $\Theta_{n}(x)$ is defined by Theorem 4.3.

Proof Let $\psi \in C_{B}^{2}\left(\mathbb{R}^{+}\right)$; for all $\varphi \in(x, t)$ a Taylor series expansion is

$$
\psi(t)=\frac{(t-x)^{2}}{2} \psi^{\prime \prime}(\varphi)+(t-x) \psi^{\prime}(x)+\psi(x) .
$$

On applying $L_{n, \alpha}^{*}$, using linearity,

$$
L_{n, \alpha}^{*}(\psi ; x)-\psi(x)=\psi^{\prime}(x) L_{n, \alpha}^{*}((t-x) ; x)+\frac{\psi^{\prime \prime}(\varphi)}{2} L_{n, \alpha}^{*}\left((t-x)^{2} ; x\right),
$$

which implies that

$$
\begin{aligned}
& \left|L_{n, \alpha}^{*}(\psi ; x)-\psi(x)\right| \\
& \leq \leq\left(\frac{2 \alpha-1}{n-1} x+\frac{1}{n-1}\right)\left\|\psi^{\prime}\right\|_{C_{B}[0, \infty)} \\
& \quad+\left\{\frac{2 n+2(4 \alpha-3)}{(n-2)(n-1)} x^{2}+\frac{2 n+2(5 \alpha-3)}{(n-2)(n-1)} x+\frac{2}{(n-2)(n-1)}\right\} \frac{\left\|\psi^{\prime \prime}\right\|_{C_{B}[0, \infty)}}{2} .
\end{aligned}
$$

From (4.3) we have $\left\|\psi^{\prime}\right\|_{C_{B}[0, \infty)} \leq\|\psi\|_{C_{B}^{2}[0, \infty)},\left\|\psi^{\prime \prime}\right\|_{C_{B}[0, \infty)} \leq\|\psi\|_{C_{B}^{2}[0, \infty)}$.

$$
\begin{aligned}
& \left|L_{n, \alpha}^{*}(\psi ; x)-\psi(x)\right| \\
& \leq \leq\left(\frac{2 \alpha-1}{n-1} x+\frac{1}{n-1}\right)\|\psi\|_{C_{B}^{2}[0, \infty)} \\
& \quad+\left\{\frac{2 n+2(4 \alpha-3)}{(n-2)(n-1)} x^{2}+\frac{2 n+2(5 \alpha-3)}{(n-2)(n-1)} x+\frac{2}{(n-2)(n-1)}\right\} \frac{\|\psi\|_{[0, \infty)}}{2} .
\end{aligned}
$$

This completes the proof.

In 1968 [34] for investigating the interpolation between two Banach spaces Peetre introduced the $K$-functional by

$$
K_{2}(f ; \delta)=\inf _{C_{B}^{2}[0, \infty)}\left\{\left(\|f-\psi\|_{C_{B}[0, \infty)}+\delta\|\psi\|_{C_{B}^{2}[0, \infty)}\right): \psi \in C_{B}^{2}[0, \infty)\right\}
$$

and a positive constant $\mathfrak{D}$ exists such that $K_{2}(f ; \delta) \leq \mathfrak{D} \omega_{2}\left(f ; \delta^{\frac{1}{2}}\right)$ with $\delta>0$ and $\omega_{2}(f ; \delta)$ is the second-order modulus of continuity. 
Theorem 4.8 Suppose $C_{B}[0, \infty)$ is the set of all bounded and continuous functions on $[0, \infty)$. Then for every $f \in C_{B}[0, \infty)$

$$
\left|L_{n, \alpha}^{*}(f ; x)-f(x)\right| \leq 2 \mathfrak{D}\left\{\omega_{2}\left(f ; \sqrt{\mathfrak{K}_{n}(x)}\right)+\min \left(1, \mathfrak{K}_{n}(x)\right)\|f\|_{C_{B}[0, \infty)}\right\},
$$

where $\mathfrak{K}_{n}(x)=\frac{2 \Delta_{n}(x)+\Theta_{n}(x)}{4}$ is defined by Theorem 4.7 .

Proof In the light of results obtained by Theorem 4.7, we prove the desired theorem; hence

$$
\begin{aligned}
\left|L_{n, \alpha}^{*}(f ; x)-f(x)\right| & \leq\left|L_{n, \alpha}^{*}(f-\psi ; x)\right|+|f(x)-\psi(x)|+\left|L_{n, \alpha}^{*}(\psi ; x)-\psi(x)\right| \\
& \leq 2\|f-\psi\|_{C_{B}[0, \infty)}+\left(\frac{\Theta_{n}(x)}{2}+\Delta_{n}(x)\right)\|\psi\|_{C_{B}^{2}[0, \infty)} \\
& =2\left(\|f-\psi\|_{C_{B}[0, \infty)}+\left(\frac{\Theta_{n}(x)}{4}+\frac{\Delta_{n}(x)}{2}\right)\|\psi\|_{C_{B}^{2}[0, \infty)}\right) .
\end{aligned}
$$

If we take the infimum over all $\psi \in C_{B}^{2}[0, \infty)$ and we use (4.11), we get

$$
\left|L_{n, \alpha}^{*}(f ; x)-f(x)\right| \leq 2 K_{2}\left(f ;\left(\frac{\Theta_{n}(x)}{4}+\frac{\Delta_{n}(x)}{2}\right)\right) .
$$

Now from [35] we use the relation for an absolute constant $\mathfrak{D}>0$

$$
K_{2}(f ; \delta) \leq \mathfrak{D}\left\{\omega_{2}(f ; \sqrt{\delta})+\min (1, \delta)\|f\|\right\} .
$$

This completes the proof.

\section{Conclusion and observations}

The manuscript parametric variant of Baskakov-Durrmeyer operators is a new extension of Baskakov Durrmeyer type operators. In the present investigation in our manuscript in order to get uniform convergence for the operators of the $\alpha$-type extended version we study the order of approximation, the rate of convergence, the Korovkin-type, the weighted Korovkin-type approximation theorems, Peetres $K$-functional, Lipschitz functions and a set of direct theorems. It must be noted that we have more modeling flexibility when adding the parameter $\alpha$ to the Baskakov-Durrmeyer operators.

Funding

Not applicable.

Competing interests

All authors of this manuscript declare that they have no competing interests.

Authors' contributions

All authors have read the manuscript and ensure that they approve of all its content as regards integrity and accuracy of their accountability.

\section{Author details}

${ }^{1}$ Department of Computer Science (SEST), Jamia Hamdard University, New Delhi, India. ${ }^{2}$ Department of Mathematics, Jamia Millia Islamia University, New Delhi, India. ${ }^{3}$ Post Graduate Department of Mathematics, Patna University, Patna, India.

\section{Publisher's Note}

Springer Nature remains neutral with regard to jurisdictional claims in published maps and institutional affiliations. 


\section{References}

1. Bernstein, S.N.: Démonstration du Théorème de Weierstrass fondée sur le calcul de probabilités. Commun. Soc. Math. Kharkow 13(2), 1-2 (1912-1913)

2. Acar, T., Aral, A., Mohiuddine, S.A.: On Kantorovich modification of (p,q)-Baskakov operators. J. Inequal. Appl. 2016, Article ID 98 (2016)

3. Acar, T., Agrawal, P.N., Kumar, A.S.: On a modification of (p,q)-Szász-Mirakyan operators. Complex Anal. Oper. Theory 12(1), 155-167 (2018)

4. Acar, T., Aral, A., Mohiuddine, S.A.: On Kantorovich modification of $(p, q)$-Bernstein operators. Iran. J. Sci. Technol., Trans. A, Sci. 42, 1459-1464 (2018)

5. Acar, T., Mursaleen, M., Mohiuddine, S.A.: Stancu type (p,q)-Szász-Mirakyan-Baskakov operators. Commun. Fac. Sci. Univ. Ank. Sér. A1 Math. Stat. 67(1), 116-128 (2018)

6. Acar, T., Aral, A., Mohiuddine, S.A.: Approximation by bivariate $(p, q)$-Bernstein-Kantorovich operators. Iran. J. Sci. Technol., Trans. A, Sci. 42, 655-662 (2018)

7. Acar, T., Mohiuddine, S.A., Mursaleen, M.: Approximation by $(p, q)$-Baskakov-Durrmeyer-Stancu operators. Complex Anal. Oper. Theory 12, 1453-1468 (2018)

8. Acu, A.M., Muraru, C.V.: Approximation properties of bivariate extension of $q$-Bernstein-Schurer-Kantorovich operators. Results Math. 67(3-4), 265-279 (2015)

9. Acu, A.M.: Stancu-Schurer-Kantorovich operators based on q-integers. Appl. Math. Comput. 259, 896-907 (2015)

10. Bărbosu, D.: Some applications of Shisha-Mond theorem. Creative Math. Inform. 23(2), 141-146 (2014)

11. Agrawal, P.N., Kumar, D., Araci, S.: Linking of Bernstein-Chlodowsky and Szász-Appell-Kantorovich type operators. J. Nonlinear Sci. Appl. 10(6), 3288-3302 (2017)

12. Aral, A., Acar, T.: Weighted approximation by new Bernstein-Chlodowsky-Gadjiev operators. Filomat 27(2), 371-380 (2013)

13. Mursaleen, M., Ansari, K.J., Khan, A.: On $(p, q)$-analogue of Bernstein operators. Appl. Math. Comput. 266, 874-882 (2015) \& [MR3377604], Appl. Math. Comput. 278, 70-71 (2016)

14. Mursaleen, M., Nasiruzzaman, M., Nurgali, A.: Some approximation results on Bernstein-Schurer operators defined by (p, q)-integers. J. Inequal. Appl. 2015, Article ID 249 (2015)

15. Mursaleen, M., Khan, F., Khan, A.: Approximation by Kantorovich type q-Bernstein-Stancu operators. Complex Anal. Oper. Theory 11(1), 85-107 (2017)

16. Khan, A., Sharma, V.: Statistical approximation by $(p, q)$-analogue of Bernstein-Stancu operators. Azerb. J. Math. 8(2), 100-121 (2018)

17. Alotaibi, A., Nasiruzzaman, M., Mursaleen, M.: A Dunkl type generalization of Szász operators via post-quantum calculus. J. Inequal. Appl. 2018, Article ID 287 (2018)

18. Srivastava, H.M., Jena, B.B., Paikray, S.K., Misra, U.K.: A certain class of weighted statistical convergence and associated Korovkin-type approximation theorems involving trigonometric functions. Math. Methods Appl. Sci. 41, 671-683 (2018)

19. Srivastava, H.M., Mursaleen, M., Alotaibi, A., Nasiruzzaman, M., Al-Abied, A.: Some approximation results involving the q-Szász-Mirakjan-Kantorovich type operators via Dunkl's generalization. Math. Methods Appl. Sci. 40, 5437-5452 (2017)

20. Srivastava, H.M., Özger, F., Mohiuddine, S.A.: Construction of Stancu-type Bernstein operators based on Bézier bases with shape parameter $\lambda$. Symmetry 11, 316 (2019). https://doi.org/10.3390/sym11030316

21. Wafi, A., Rao, N.: Szász-Durrmeyer operators based on Dunkl analogue. Complex Anal. Oper. Theory (2018). https://doi.org/10.1007/s11785-017-0647-7

22. Rao, N., Wafi, A., Acu, A.M.: q-Szász-Durrmeyer type operators based on Dunkl analogue. Complex Anal. Oper. Theory (2018). https://doi.org/10.1007/s11785-018-0816-3

23. Kadak, U.: On weighted statistical convergence based on $(p, q)$-integers and related approyimation theorems for functions of two variables. J. Math. Anal. Appl. 443(2), 752-764 (2016)

24. Kadak, U.: Weighted statistical convergence based on generalized difference operator involving $(p, q)$-gamma function and its applications to approximation theorems. J. Math. Anal. Appl. 448(2), 1633-1650 (2017)

25. Milovanovic, G.V., Mursaleen, M., Nasiruzzaman, M.: Modified Stancu type Dunkl generalization of Szász-Kantorovich operators. Rev. R. Acad. Cienc. Exactas Fís. Nat., Ser. A Mat. 112, 135-151 (2018)

26. Mohiuddine, S.A., Acar, T., Alotaibi, A.: Construction of a new family of Bernstein-Kantorovich operators. Math. Methods Appl. Sci. 40, 7749-7759 (2017)

27. Mohiuddine, S.A., Acar, T., Alotaibi, A.: Durrmeyer type $(p, q)$-Baskakov operators preserving linear functions. J. Math. Inequal. 12, 961-973 (2018)

28. Mohiuddine, S.A., Acar, T., Alghamdi, M.A.: Genuine modified Bernstein-Durrmeyer operators. J. Inequal. Appl. 2018, Article ID 104 (2018)

29. Kadak, U., Mohiuddine, S.A.: Generalized statistically almost convergence based on the difference operator which includes the $(p, q)$-gamma function and related approximation theorems. Results Math. 73, Article 9 (2018)

30. Edely, H., Mohiuddine, S.A., Noman, A.K.: Korovkin type approximation theorems obtained through generalized statistical convergence. Appl. Math. Lett. 23, 1382-1387 (2010)

31. Cai, Q., Lian, B., Zhou, G.: Approximation properties of $\lambda$-Bernstein operators. J. Inequal. Appl. 2018, Article ID 61 (2018). https://doi.org/10.1186/s13660-018-1653-7

32. Ali, A., Erbay, H.: Parametric generalization of Baskakov operaors. Math. Commun. 24, 119-131 (2019)

33. Erencin, A.: Durrmeyer type modification of generalized Baskakov operators. Appl. Math. Comput. 218(3), 4384-4390 (2011)

34. Peetre, J.: A Theory of Interpolation of Normed Spaces. Noteas de Mathematica, vol. 39. Instituto de Mathemática Pura e Applicada, Conselho Nacional de Pesquidas, Rio de Janeiro (1968)

35. Ciupa, A.: A class of integral Favard-Szász type operators. Stud. Univ. Babeş-Bolyai, Math. 40(1), 39-47 (1995) 